\title{
Uwe Hassler (2007): Stochastische Integration und Zeitreihenmodellierung
}

\author{
Eine Einführung mit Anwendungen aus Finanzierung und \\ Ökonometrie Springer, xvi + 325 pp., Softcover, $€ 32.95$, \\ ISBN: 978-3-540-73567-0
}

\section{Christoph Hanck}

Published online: 7 June 2008

(C) The Author(s) 2008

This is a very careful introduction to time series modeling and stochastic integration. The techniques are applied to topics from finance (e.g., continuous-time interest rate models) as well as macroeconometrics (e.g., cointegration). The author fully succeeds at effectively developing the common ground of these seemingly disparate fields. As claimed on the back cover, the book is indeed "intuitive yet rigorous". The author avoids inessential generalizations (e.g., zero initial conditions are assumed where they could be stationary), which allows him to provide clear and transparent proofs of most results. Whenever topics are not discussed in full detail, references to further reading are provided.

The likely readership are advanced undergraduate students majoring in finance or econometrics. The volume can also serve as textbook for a graduate level topics course. It will also help applied researchers gain a deeper understanding of the theoretical background underlying widely applied techniques. The prerequisites are (a firm understanding of) undergraduate mathematics and statistical inference, as provided in first and second year economics courses. Familiarity with regression is sometimes helpful.

Proofs and technical arguments are relegated to the exercises. This makes the main text very readable. Detailed solutions are provided, so that these important aspects can also be thoroughly appreciated by students (and makes the text great for self-tuition). A drawback is, of course, that these exercises are no longer available for classroom use. Alternatively, proofs could have been collected in appendices, making room for "genuine" exercises. These could comprise empirical applications, which are missing in the text. Especially with less technically minded audiences, these would likely have to be supplied from other sources to maintain students' motivation.

C. Hanck ( $\square)$

TU Dortmund, Dortmund, Germany

e-mail: christoph.hanck@uni-dortmund.de 
Here is a more detailed summary: Chap. 1 is an appetizer. Chapter 2 provides a concise review of some probabilistic background, such as random variables, moments and joint distributions. This makes the treatment quite self-contained. Maybe, the material could have been slightly more focussed on the subsequent needs. If, for instance, it is anticipated that the definition of an expectation should be recalled to the students, then it may also be helpful to state properties such as linearity, which are used routinely later on. Chapter 3 discusses general linear $\left(e_{t}=\sum_{j=0}^{\infty} c_{j} \epsilon_{t-j}\right)$ and ARMA processes, deriving moments and conditions for invertibility and stationarity. The treatment is particularly clear as regards the purpose of summability conditions of the coefficient sequences, $\sum_{j=0}^{\infty}\left|c_{j}\right|<\infty$. Since much of the later material requires one-summability, $\sum_{j=0}^{\infty} j\left|c_{j}\right|<\infty$, this condition and its implications might have already been introduced here. Chapter 4 presents a remarkably accessible introduction to frequency domain time series analysis. The focus is on simple cases, such as the spectrum of an ARMA(1,1). Arguably, better use of this investment could have been made in the sequel. Chapter 5 introduces processes with conditional heteroscedasticity, i.e. (G)ARCH and some of its many offsprings like EGARCH.

Chapter 6 begins the discussion of Wiener processes $W(t)$, starting with an intuitive heuristic presentation. Properties as non-differentiability and related processes such as the Brownian Bridge $W(t)-t W(1)$, along with the corresponding moments, are introduced. Chapters 7-9 provide the requisite integration theory, skilfully proceeding from the "easier" Riemann integral $\int_{0}^{t} f(s) W(s) \mathrm{d} s$ through the Stieltjes integral $\int_{0}^{t} f(s) \mathrm{d} W(s)$ to the Ito integral, e.g., $\int_{0}^{t} f(s) W(s) \mathrm{d} W(s)$. Uwe Hassler strikes an appropriate balance between providing the right level of rigour and avoiding inessential technicalities. Of interest in its own right as well as for later use, the chapters derive the distribution of important stochastic integrals such as $\int_{0}^{t} W(s) \mathrm{d} s$. Chapter 10 is devoted to Ito's Lemma in both the univariate and multivariate case. Many examples illustrate the Lemma. Proceeding from applying it to a simple function of $W(t)$ to more general diffusions such as the Ornstein-Uhlenbeck process will undoubtedly help the reader master the ideas conveyed here. Chapter 11 exploits Ito's Lemma to the solution of general stochastic differential equations (SDEs). The focus on linear SDEs, both with constant and time-varying coefficients, yields interpretable closed-form solutions. The brief exploration of numerical simulation of SDEs is useful. Chapter 12 applies the previous results to popular continuous-time economic interest rate models, e.g., those of Vasicek and Cox, Ingersoll and Ross.

Chapter 13 begins the analysis of limiting distributions of estimators and test statistics when integrated $(I(1))$-processes are involved. It provides a general functional central limit theorem (or "invariance principle"). This ensures that properly scaled partial sums of $I(0)$-processes such as a white noise $\left\{\epsilon_{t}\right\}$ converge to a Wiener process as the number of observations diverges, $n^{-1 / 2} \sum_{j=1}^{[s n]} \epsilon_{j} \Rightarrow W(s)$. The role of the one-summability condition on the $I(0)$-process could have been clarified by introducing the Beveridge and Nelson (1981) decomposition. Teamed with the Continuous Mapping Theorem, limiting expressions for the quantities of interest are then derived efficiently. For example, $n^{-1} \sum_{t=1}^{n} n^{-1 / 2} \sum_{j=1}^{t} \epsilon_{j} \Rightarrow \int_{0}^{1} W(s) \mathrm{d} s$. The corresponding limit theory for multivariate processes prepares the ground for the analysis of cointegration. The author also gives a flavor of the underlying theory of weak convergence, 
without distracting the reader with mathematical subtleties. Chapters 14 and 15 reap the benefits of these investments, deriving the limiting distributions of popular unit root (e.g., Dickey and Fuller's 1979) and cointegration tests as functionals of Wiener processes. Also, the asymptotic distributions of the least squares estimator in trend, spurious and cointegrating regressions are presented.

As is most likely clear, the reviewer finds this book to be highly useful. Are there, nevertheless, features missing? Yes — though most are rather desiderata for a possible next, extended edition than genuine flaws. For instance, the book has little to say on estimation. Also, at little extra cost, Uwe Hassler could have further spelled out some of the idiosyncracies of unit root econometrics. Having derived the distribution of some test statistic, it would have been easy to supply critical values. Similarly, plotting the test statistics' distributions (and, along the way, explaining how to simulate these) to highlight their location and skewness would have been straightforward and yet likely illuminating. Also, having introduced the Ornstein-Uhlenbeck process, it would have been relatively easy to cover local-to-unity asymptotics (Phillips 1987), an often neglected important topic. As for cointegration, some relatively standard material, such as the Johansen (1988) methodology or the Engle and Granger (1987) non-cointegration test, is missing. For practical purposes, a word of caution on finite sample considerations might have been helpful.

To summarize, the author has fully succeeded at delivering a textbook which hits just the right note for the intended audience and will prove valuable in helping a wide readership gain access to fascinating and still rapidly evolving literatures!

Open Access This article is distributed under the terms of the Creative Commons Attribution Noncommercial License which permits any noncommercial use, distribution, and reproduction in any medium, provided the original author(s) and source are credited.

\section{References}

Beveridge S, Nelson CR (1981) A new approach to decomposition of economic time series into permanent and transitory components with particular attention to measurement of the business cycle. J Monet Econ 7:151-174

Dickey DA, Fuller WA (1979) Distribution of the estimators for autoregressive time series with a unit root. J Am Stat Assoc 74:427-431

Engle R, Granger C (1987) Co-integration and error correction: representation, estimation, and testing. Econometrica 55:251-76

Johansen S (1988) Statistical analysis of cointegration vectors. J Econ Dyn Control 12:231-254

Phillips PCB (1987) Towards a unified asymptotic theory for autoregression. Biometrika 74:535-547 